\title{
OTRA Based Second Order Universal Filter and its optimization like Butterworth, Chebyshev and Bessel
}

\author{
Vivek Bhatt ${ }^{1}$, Pramod Benjwal ${ }^{2}$ and Manoj Joshi ${ }^{3}$ \\ Department of Electronics and communication Engineering, \\ Institute Of Technology, Gopeshwar Uttarakhand, India \\ vivekbhatt1987@gmail.com,pramodben@gmail.com,manojjoshi1506@gmail.com
}

\begin{abstract}
This research work brings operational transresistance amplifier (OTRA) based second order universal filter, with three main filter optimizations like Butterworth, Chebyshev and Bessel. The universal filter offers all five sections of filter responses likes: Low-Pass, High-Pass, Band-Pass, BandRejection and All-Pass. Design is based on the RC-RC decomposition technique. Finally, simulation results are performed to verify the theoretical results using ORCAD 10.5 circuit simulator.
\end{abstract}

Index Terms-Operational Transresistance Amplifiers (OTRA), Second Order Universal Filter, Butterworth, Chebyshev and Bessel Filter.

\section{INTRODUCTION}

A number of voltage-mode (VM) and current-mode (CM) filtering circuits have been designed using different active elements such as operational amplifiers [1], operational transconductance amplifiers [2], current conveyors [3], current differencing buffered amplifier [4] etc, which have the low power consumption, high slew rate, wide bandwidth etc. Now days the operational transresistance amplifier (OTRA) has acts as an substitutional analog building block [5] that posses all the advantages of current mode techniques. OTRA eliminate parasitic capacitances throw input grounded terminals[6-8]. Presently OTRA manufactured commercially as norton amplifiers or differencing amplifiers for several integrated circuit. Manufactures observed that CMOS OTRA is more simpler and more efficient than the commercially available another building blocks. Other useful applications of OTRA have been reported in literature [9-11]. These reports include, designing of universal filter [9], single- resistancecontrolled oscillators [10], and all-pass filters [11]. On the other hand, a literature study enhance our knowledge that both Kerwin-Huelsman-Newcomb (KHN) and Tow-Thomas (TT) biquads designed by using current conveyors, operational amplifier or OTAs [12-15]. On the basis of literature study we found that although Fleischer-Tow biquad, is an improved version of the Tow-Thomas configuration, offers the realization of all five different second-order filtering functions, namely low-pass, high-pass, band-pass, notch, and all-pass. But as per the author knowledge not a single paper that includes all filter optimization like Butterworth, Chebyshev and Bessel are reported in a single paper.

The purpose of this work is to analyse second order universal filter structure with its all optimizations like Butterworth,
Chebyshev and Bessel, that exhibits all responses of filter like Band-Pass (BP), Band-Reject (BR), Low-Pass (LP), HighPass (HP) and All-Pass (AP) functions from the same configuration. Also, a comparative study for the second order universal filter is reported in this paper.

\section{CIRCUIT DESCRIPTION}

A $0.5 \mu \mathrm{m}$ Complementary metal oxide semiconductor technology based internal circuit of operational transresistance amplifier is shown in Figure 1 [5], and the schematic circuit symbol of OTRA is shown in Figure 2. Corresponding input/ output - relationship is characterized as:

$$
\mathrm{V}_{\mathrm{p}}=\mathrm{V}_{\mathrm{n}}=0 \text { and } \mathrm{V}_{0}=\mathrm{R}_{\mathrm{m}}\left(\mathrm{I}_{\mathrm{p}}-\mathrm{I}_{\mathrm{n}}\right)
$$

where, $V_{p}$ and $V_{n}$ is the input voltage respectively at terminal $p$ and $n$, with the transresistance gain $R_{m}$ which approaches infinity for ideal one.

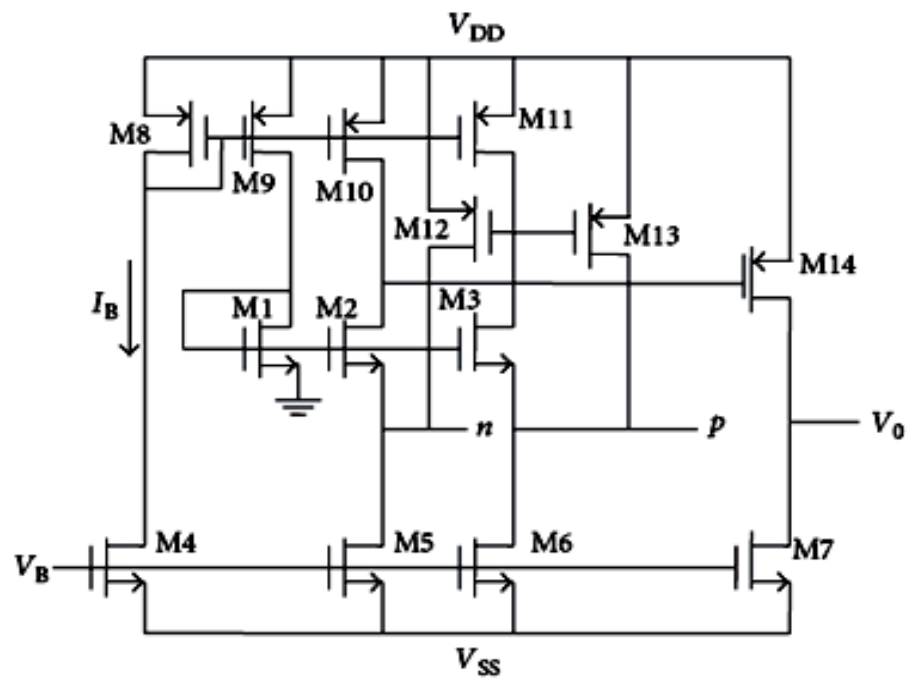

Fig. 1. Internal circuit of OTRA [5]

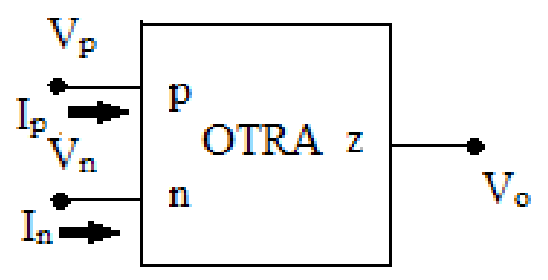

Fig. 2. The schematic circuit symbol of an OTRA 
The proposed work uses generalized filter structure using eight admittance term shown in Figure 3. The corresponding transfer function may be obtained by the routine analysis as:

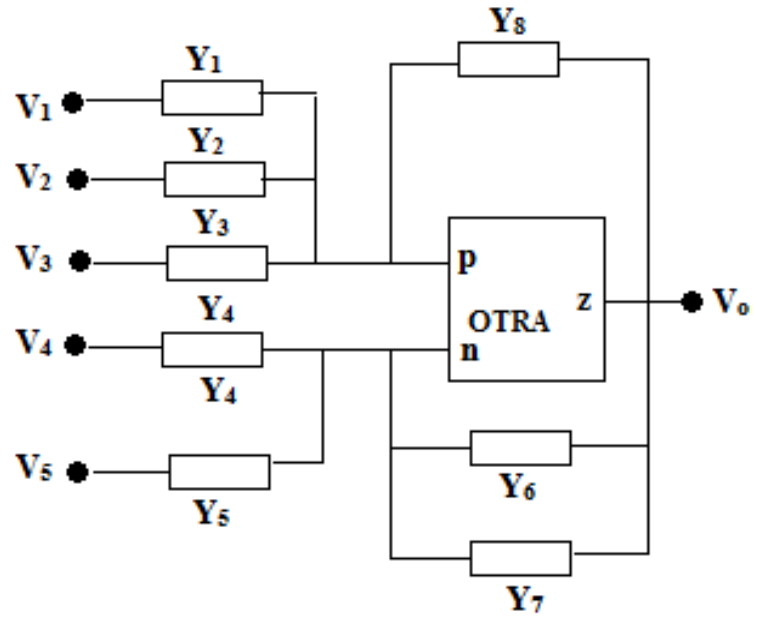

Fig.3. Generalized OTRA based filter structure

$$
\frac{\mathrm{N}(\mathrm{s})}{\mathrm{D}(\mathrm{s})}=\frac{\mathrm{Y}_{1} \mathrm{~V}_{1}+\mathrm{Y}_{2} \mathrm{~V}_{2}+\mathrm{Y}_{3} \mathrm{~V}_{3}-\mathrm{Y}_{4} \mathrm{~V}_{4}-\mathrm{Y}_{5} \mathrm{~V} 5}{\mathrm{Y}_{6}+\mathrm{Y}_{7}-\mathrm{Y}_{8}}
$$

where, $\mathrm{Y}_{1}, \mathrm{Y}_{2}, \mathrm{Y}_{3} \mathrm{Y}_{4}, \mathrm{Y}_{5}, \mathrm{Y}_{6}, \mathrm{Y}_{7}$ and $\mathrm{Y}_{8}$ are positive admittance terms. The generalized structure can be used for design different type of filter optimization like Butterworth, Chebyshev and Bessel filter. The general transfer function of second order low-pass filter can be expressed as:

$$
\mathrm{T}(\mathrm{s})=\frac{\mathrm{A}_{0}}{\left(1+\mathrm{A}_{1} \mathrm{~s}+\mathrm{B}_{1} \mathrm{~s}^{2}\right)}
$$

Here the design part is done with unity gain $\left(A_{0}=1\right)$ and the filter coefficients $A_{1}$ and $B_{1}$ must be different for different types of filter transfer function, which is given in Table 1.

TABLE. 1

SECOND ORDER FILTER COEFFICIENTS

\begin{tabular}{|c|c|}
\hline Types of Filter & Filter Coefficients \\
\hline $\begin{array}{c}\text { Butterworth } \\
\text { Filter }\end{array}$ & $\mathrm{A}_{1}=1.4142$ \\
& $\mathrm{~B}_{1}=1.0000$ \\
\hline Chebyshev Filter & $\mathrm{A}_{1}=1.3022$ \\
& $\mathrm{~B}_{1}=1.5515$ \\
\hline \multirow{2}{*}{ Bessel Filter } & $\mathrm{A}_{1}=1.3617$ \\
& $\mathrm{~B}_{1}=0.6180$ \\
\hline
\end{tabular}

The transfer function is decomposing by RC-RC decomposition technique [14] as below:

The general transfer function of second order low pass filter is

$$
\mathrm{T}(\mathrm{s})=\frac{\mathrm{A}_{0}}{\left(1+\mathrm{A}_{1} \mathrm{~s}+\mathrm{B}_{1} \mathrm{~s}^{2}\right)}
$$

Rearrange the equation as [14]:

$$
\mathrm{T}(\mathrm{s})=\frac{\mathrm{N}(\mathrm{s})}{\mathrm{D}(\mathrm{s})}=\frac{\frac{1}{(\mathrm{~s}+1)}}{\frac{\mathrm{D}(\mathrm{s})}{(\mathrm{s}+1)}}
$$

There after second order low pass Butterworth filter having numerator and denominator must be decomposed as [14]

$$
\begin{aligned}
& \mathrm{N}(\mathrm{s})=\frac{1}{(\mathrm{~s}+1)}=-\frac{\mathrm{s}}{(\mathrm{s}+1)}+1 \\
& \mathrm{D}(\mathrm{s})=\frac{1+1.4142 \mathrm{~s}+1.0000 \mathrm{~s}^{2}}{(\mathrm{~s}+1)} \\
& =\mathrm{s}+0.4142+\frac{0.5858}{(\mathrm{~s}+1)} \\
& =\mathrm{s}-\frac{0.5858 \mathrm{~s}}{(\mathrm{~s}+1)}+1
\end{aligned}
$$

Similarly for second order low pass chebyshev having numerator and denominator must be decomposed as [14]

$$
\begin{aligned}
& \mathrm{N}(\mathrm{s})=\frac{1}{(\mathrm{~s}+1)}=-\frac{\mathrm{s}}{(\mathrm{s}+1)}+1 \\
& \mathrm{D}(\mathrm{s})=\frac{1+1.3022 \mathrm{~s}+1.5515 \mathrm{~s}^{2}}{(\mathrm{~s}+1)} \\
& =1.5515 \mathrm{~s}+0.2493+\frac{1.2493}{(\mathrm{~s}+1)} \\
& =1.5515 \mathrm{~s}-\frac{1.2493 \mathrm{~s}}{(\mathrm{~s}+1)}+1
\end{aligned}
$$

Again for second order low pass Bessel filter having numerator and denominator must be decomposed as [14]

$$
\begin{aligned}
& N(s)=\frac{1}{(s+1)}=-\frac{s}{(s+1)}+1 \\
& D(s)=\frac{1+1.3617 s+0.6180 s^{2}}{(s+1)} \\
& =0.6180 s+0.7437+\frac{0.2563}{(s+1)} \\
& =0.6180 s-\frac{0.2563 s}{(s+1)}+1
\end{aligned}
$$

Finally, all other filter response like high pass, band pass, band reject and all pass response like Butterworth, Chebyshev and Bessel filters are summarized with their transfer function and decomposed as per the above discussion which is summarized in Table 2. 
TABLE 2. TRANSFER FUNCTION DECOMPOSITION

\begin{tabular}{|c|c|c|}
\hline Optimization & Types & Transfer function Decomposition \\
\hline \multirow{5}{*}{ Butterworth } & $\begin{array}{l}\text { Low } \\
\text { pass }\end{array}$ & $\mathrm{N}(\mathrm{s})=\frac{1}{(\mathrm{~s}+1)}=-\frac{\mathrm{s}}{(\mathrm{s}+1)}+1, \mathrm{D}(\mathrm{s})=\frac{1+1.4142 \mathrm{~s}+1.0000 \mathrm{~s}^{2}}{(\mathrm{~s}+1)}=\mathrm{s}-\frac{0.5858 \mathrm{~s}}{(\mathrm{~s}+1)}+1$ \\
\hline & $\begin{array}{l}\text { High } \\
\text { pass }\end{array}$ & $\mathrm{N}(\mathrm{s})=\frac{\mathrm{s}^{2}}{(\mathrm{~s}+1)}=\mathrm{s}-1+\frac{\mathrm{s}}{(\mathrm{s}+1)}, \mathrm{D}(\mathrm{s})=\frac{1+1.4142 \mathrm{~s}+1.0000 \mathrm{~s}^{2}}{(\mathrm{~s}+1)}=\mathrm{s}-\frac{0.5858 \mathrm{~s}}{(\mathrm{~s}+1)}+1$ \\
\hline & $\begin{array}{l}\text { Band } \\
\text { pass }\end{array}$ & $\mathrm{N}(\mathrm{s})=\frac{\mathrm{s}}{(\mathrm{s}+1)}=\frac{\mathrm{s}}{(\mathrm{s}+1)}+1-1, \mathrm{D}(\mathrm{s})=\frac{1+1.4142 \mathrm{~s}+1.0000 \mathrm{~s}^{2}}{(\mathrm{~s}+1)}=\mathrm{s}-\frac{0.5858 \mathrm{~s}}{(\mathrm{~s}+1)}+1$ \\
\hline & $\begin{array}{l}\text { Band } \\
\text { reject }\end{array}$ & $\mathrm{N}(\mathrm{s})=\frac{\mathrm{s}^{2}+1}{(\mathrm{~s}+1)}=\mathrm{s}+1-\frac{2 \mathrm{~s}}{(\mathrm{~s}+1)} \mathrm{D}(\mathrm{s})=\frac{1+1.4142 \mathrm{~s}+1.0000 \mathrm{~s}^{2}}{(\mathrm{~s}+1)}=\mathrm{s}-\frac{0.5858 \mathrm{~s}}{(\mathrm{~s}+1)}+1$ \\
\hline & $\begin{array}{l}\text { All } \\
\text { pass }\end{array}$ & $\begin{array}{l}\mathrm{N}(\mathrm{s})=\frac{1-1.4142 \mathrm{~s}+1.0000 \mathrm{~s}^{2}}{(\mathrm{~s}+1)}=\mathrm{s}+1-\frac{3.4142 \mathrm{~s}}{(\mathrm{~s}+1)}, \mathrm{D}(\mathrm{s})=\frac{1+1.4142 \mathrm{~s}+1.0000 \mathrm{~s}^{2}}{(\mathrm{~s}+1)} \\
\mathrm{s}-\frac{0.5858 \mathrm{~s}}{(\mathrm{~s}+1)}+1\end{array}$ \\
\hline \multirow{5}{*}{ Chebyshev } & $\begin{array}{l}\text { Low } \\
\text { pass }\end{array}$ & $\mathrm{N}(\mathrm{s})=\frac{1}{(\mathrm{~s}+1)}=-\frac{\mathrm{s}}{(\mathrm{s}+1)}+1, \mathrm{D}(\mathrm{s})=\frac{1+1.3022 \mathrm{~s}+1.5515 \mathrm{~s}^{2}}{(\mathrm{~s}+1)}=1.5515 \mathrm{~s}-\frac{1.2493 \mathrm{~s}}{(\mathrm{~s}+1)}+1$ \\
\hline & $\begin{array}{l}\text { High } \\
\text { pass }\end{array}$ & $\mathrm{N}(\mathrm{s})=\frac{\mathrm{s}^{2}}{(\mathrm{~s}+1)}=\mathrm{s}-1+\frac{\mathrm{s}}{(\mathrm{s}+1)}, \mathrm{D}(\mathrm{s})=\frac{1+1.3022 \mathrm{~s}+1.5515 \mathrm{~s}^{2}}{(\mathrm{~s}+1)}=1.5515 \mathrm{~s}-\frac{1.2493 \mathrm{~s}}{(\mathrm{~s}+1)}+1$ \\
\hline & $\begin{array}{l}\text { Band } \\
\text { pass }\end{array}$ & $\mathrm{N}(\mathrm{s})=\frac{\mathrm{s}}{(\mathrm{s}+1)}=\frac{\mathrm{s}}{(\mathrm{s}+1)}+1-1, \mathrm{D}(\mathrm{s})=\frac{1+1.3022 \mathrm{~s}+1.5515 \mathrm{~s}^{2}}{(\mathrm{~s}+1)}=1.5515 \mathrm{~s}-\frac{1.2493 \mathrm{~s}}{(\mathrm{~s}+1)}+1$ \\
\hline & $\begin{array}{l}\text { Band } \\
\text { reject }\end{array}$ & $\mathrm{N}(\mathrm{s})=\frac{\mathrm{s}^{2}+1}{(\mathrm{~s}+1)}=\mathrm{s}+1-\frac{2 \mathrm{~s}}{(\mathrm{~s}+1)} \mathrm{D}(\mathrm{s})=\frac{1+1.3022 \mathrm{~s}+1.5515 \mathrm{~s}^{2}}{(\mathrm{~s}+1)}=1.5515 \mathrm{~s}-\frac{1.2493 \mathrm{~s}}{(\mathrm{~s}+1)}+1$ \\
\hline & $\begin{array}{l}\text { All } \\
\text { pass }\end{array}$ & $\begin{array}{l}\mathrm{N}(\mathrm{s})=\frac{1-1.3022 \mathrm{~s}+1.5515 \mathrm{~s}^{2}}{(\mathrm{~s}+1)}=1.5515 \mathrm{~s}+1-\frac{3.8537 \mathrm{~s}}{(\mathrm{~s}+1)}, \mathrm{D}(\mathrm{s})=\frac{1+1.3022 \mathrm{~s}+1.5515 \mathrm{~s}^{2}}{(\mathrm{~s}+1)} \\
=1.5515 \mathrm{~s}-\frac{1.2493 \mathrm{~s}}{(\mathrm{~s}+1)}+1\end{array}$ \\
\hline \multirow{5}{*}{ Bessel } & $\begin{array}{l}\text { Low } \\
\text { pass }\end{array}$ & $\mathrm{N}(\mathrm{s})=\frac{1}{(\mathrm{~s}+1)}=-\frac{\mathrm{s}}{(\mathrm{s}+1)}+1, \mathrm{D}(\mathrm{s})=\frac{1+1.3617 \mathrm{~s}+0.6180 \mathrm{~s}^{2}}{(\mathrm{~s}+1)}=0.6180 \mathrm{~s}-\frac{0.2563 \mathrm{~s}}{(\mathrm{~s}+1)}+1$ \\
\hline & $\begin{array}{l}\text { High } \\
\text { pass }\end{array}$ & $\mathrm{N}(\mathrm{s})=\frac{\mathrm{s}^{2}}{(\mathrm{~s}+1)}=\mathrm{s}-1+\frac{\mathrm{s}}{(\mathrm{s}+1)}, \mathrm{D}(\mathrm{s})=\frac{1+1.3617 \mathrm{~s}+0.6180 \mathrm{~s}^{2}}{(\mathrm{~s}+1)}=0.6180 \mathrm{~s}-\frac{0.2563 \mathrm{~s}}{(\mathrm{~s}+1)}+1$ \\
\hline & $\begin{array}{l}\text { Band } \\
\text { pass }\end{array}$ & $\mathrm{N}(\mathrm{s})=\frac{\mathrm{s}}{(\mathrm{s}+1)}=\frac{\mathrm{s}}{(\mathrm{s}+1)}+1-1, \mathrm{D}(\mathrm{s})=\frac{1+1.3617 \mathrm{~s}+0.6180 \mathrm{~s}^{2}}{(\mathrm{~s}+1)}=0.6180 \mathrm{~s}-\frac{0.2563 \mathrm{~s}}{(\mathrm{~s}+1)}+1$ \\
\hline & $\begin{array}{l}\text { Band } \\
\text { reject }\end{array}$ & $\mathrm{N}(\mathrm{s})=\frac{\mathrm{s}^{2}+1}{(\mathrm{~s}+1)}=\mathrm{s}+1-\frac{2 \mathrm{~s}}{(\mathrm{~s}+1)} \mathrm{D}(\mathrm{s})=\frac{1+1.3617 \mathrm{~s}+0.6180 \mathrm{~s}^{2}}{(\mathrm{~s}+1)}=0.6180 \mathrm{~s}-\frac{0.2563 \mathrm{~s}}{(\mathrm{~s}+1)}+1$ \\
\hline & $\begin{array}{l}\text { All } \\
\text { pass }\end{array}$ & $\begin{array}{l}\mathrm{N}(\mathrm{s})=\frac{1-1.3617 \mathrm{~s}+0.6180 \mathrm{~s}^{2}}{(\mathrm{~s}+1)}=0.6180 \mathrm{~s}+1-\frac{2.9797 \mathrm{~s}}{(\mathrm{~s}+1)} \mathrm{D}(\mathrm{s})=\frac{1+1.3617 \mathrm{~s}+0.6180 \mathrm{~s}^{2}}{(\mathrm{~s}+1)} \\
=0.6180 \mathrm{~s}-\frac{0.2563 \mathrm{~s}}{(\mathrm{~s}+1)}+1\end{array}$ \\
\hline
\end{tabular}


The obtained transfer function for Butterworth, Chebyshev and Bessel are nothing but simply the combination of $\mathrm{R}$ and $\mathrm{C}$ or simply $\mathrm{R}$ and $\mathrm{C}$. Normalized value of passive components of different optimization computed by above analysis and actual value of passive component scaled by using impedance scaling factor $(\mathrm{ZSF})=80 \times 10^{3}$ and frequency scaling factor $(\mathrm{FSF})=2 \pi \times 100 \times 10^{3}(100 \mathrm{KHz}$ desired cut off frequency), which is given in Table 3.This analysis is made with the help of paper [16].

TABLE 3: ACTUAL VALUE OF PASSIVE ELEMENTS.

\begin{tabular}{|c|c|c|c|c|c|c|c|c|c|c|c|c|}
\hline \multirow{2}{*}{$\begin{array}{c}\text { Filter } \\
\text { Responses }\end{array}$} & \multirow{2}{*}{ Types } & \multicolumn{11}{|c|}{ Component value } \\
\hline & & $\mathrm{R}_{\mathrm{a} 1}\left(\mathrm{k}^{\prime} \Omega\right)$ & $\mathrm{C}_{\mathrm{a} 1}(\mathrm{pf})$ & $\mathrm{R}_{\mathrm{a} 2}\left(\mathrm{k}^{\prime} \Omega\right)$ & $\mathrm{C}_{\mathrm{a} 2}(\mathrm{pf})$ & $\mathrm{R}_{\mathrm{b} 1}\left(\mathrm{k}^{\prime} \Omega\right)$ & $\mathrm{C}_{\mathrm{b} 1}(\mathrm{pf})$ & $\mathrm{R}_{\mathrm{b} 2}\left(\mathrm{k}^{\prime} \Omega\right)$ & $\mathrm{R}_{\mathrm{c}}(\mathrm{k} \Omega)$ & $\mathrm{C}_{\mathrm{c}}(\mathrm{pf})$ & $\mathrm{R}_{\mathrm{d}}\left(\mathrm{k}^{\prime} \Omega\right)$ & $\mathrm{C}_{\mathrm{d}}(\mathrm{pf})$ \\
\hline \multirow{5}{*}{ Butterworth } & Low pass & 80 & - & - & - & 80 & 19.89 & - & 80 & 19.89 & 136.57 & 11.65 \\
\hline & High pass & - & 19.89 & - & - & 80 & 19.89 & - & 80 & 19.89 & 136.57 & 11.65 \\
\hline & Band pass & 80 & - & 80 & 19.89 & - & - & 80 & 80 & 19.89 & 136.57 & 11.65 \\
\hline & Band reject & 80 & 19.89 & - & - & 40 & 39.78 & - & 80 & 19.89 & 136.57 & 11.65 \\
\hline & All pass & 80 & 19.89 & - & - & 23.43 & 67.91 & - & 80 & 19.89 & 136.57 & 11.65 \\
\hline \multirow{5}{*}{ Tschebyscheff } & Low pass & 80 & - & - & - & 80 & 19.89 & - & 80 & 30.85 & 64.036 & 24.85 \\
\hline & High pass & - & 19.89 & - & - & 80 & 19.89 & - & 80 & 30.85 & 64.036 & 24.85 \\
\hline & Band pass & 80 & - & 80 & 19.89 & - & - & 80 & 80 & 30.85 & 64.036 & 24.85 \\
\hline & Band reject & 80 & 19.89 & - & - & 40.76 & 39.78 & - & 80 & 30.85 & 64.036 & 24.85 \\
\hline & All pass & 80 & 30.85 & - & - & 20.76 & 39.78 & - & 80 & 30.85 & 64.036 & 24.85 \\
\hline \multirow{5}{*}{ Bessel } & Low pass & 80 & - & - & - & 80 & 19.89 & - & 80 & 12.29 & 312.13 & 5.098 \\
\hline & High pass & - & 19.89 & - & - & 80 & 19.89 & - & 80 & 12.29 & 312.13 & 5.098 \\
\hline & Band pass & 80 & - & 80 & 19.89 & - & - & 80 & 80 & 12.29 & 312.13 & 5.098 \\
\hline & Band reject & 80 & 19.89 & - & - & 40 & 39.78 & - & 80 & 12.29 & 312.13 & 5.098 \\
\hline & All pass & 80 & 12.29 & - & - & 26.85 & 59.26 & - & 80 & 12.29 & 312.13 & 5.098 \\
\hline
\end{tabular}

From above discussion we may modify the generalized equation (2) to equation (12). Here the proposed second order filter structure which is shown in Figure 3 is modified as Figure 4 and the corresponding transfer function can be written as equation (12) for Butterworth, Chebyshev and Bessel second order universal filter.

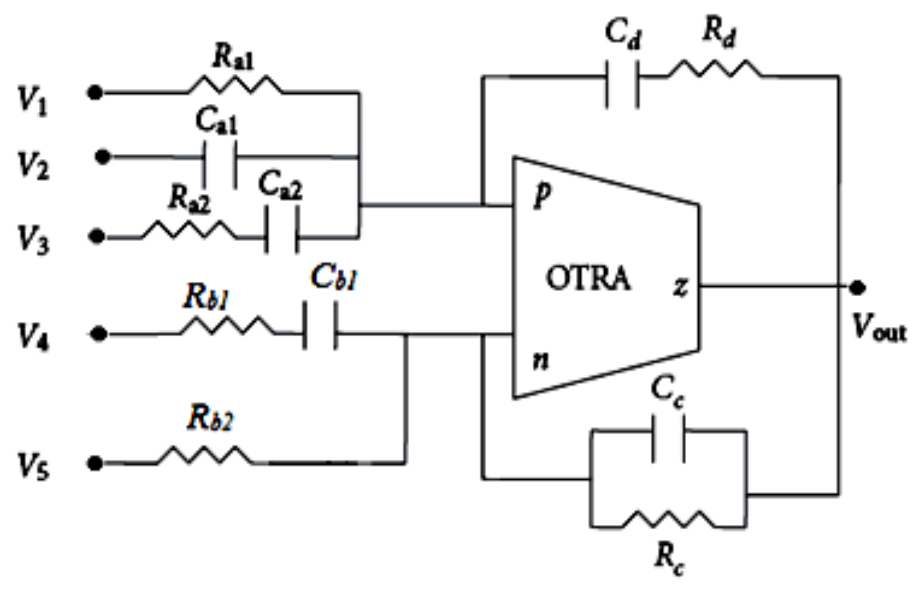

Fig.4. proposed second order universal filter $\frac{\mathrm{N}(\mathrm{s})}{\mathrm{D}(\mathrm{s})}=\frac{\frac{1}{\mathrm{R}_{\mathrm{a} 1}} \mathrm{~V}_{1}+\mathrm{C}_{\mathrm{a} 1} \mathrm{~s} \mathrm{~V}_{2}+\frac{\mathrm{C}_{\mathrm{a} 2} \mathrm{~s}}{\mathrm{R}_{\mathrm{a} 2} \mathrm{C}_{\mathrm{a} 2} \mathrm{~s}+1} \mathrm{~V}_{3}-\frac{\mathrm{C}_{\mathrm{b} 1} \mathrm{~s}}{\mathrm{R}_{\mathrm{bl}} \mathrm{C}_{\mathrm{b} 1} \mathrm{~s}+1} \mathrm{~V}_{4}-\frac{1}{\mathrm{R}_{\mathrm{b} 2}} \mathrm{~V}_{5}}{\frac{1}{\mathrm{R}_{\mathrm{c}}}+\mathrm{C}_{\mathrm{c}} \mathrm{s}-\frac{\mathrm{C}_{\mathrm{d}} \mathrm{s}}{\mathrm{R}_{\mathrm{d}} \mathrm{C}_{\mathrm{d}} \mathrm{s}+1}}$
Finally to obtain the various filter response, a proper input selection is required, Table 4 shown the corresponding selection of $\mathrm{V}_{1}, \mathrm{~V}_{2}, \mathrm{~V}_{3}, \mathrm{~V}_{4}$ and $\mathrm{V}_{5}$ to achieve different filter response.

TABLE 4. THE $\mathrm{V}_{1}, \mathrm{~V}_{2}, \mathrm{~V}_{3}, \mathrm{~V}_{4}$ AND $\mathrm{V}_{5}$ VALUES SELECTION FOR EACH FILTER FUNCTION RESPONSE

\begin{tabular}{|c|c|c|c|c|c|}
\hline $\begin{array}{c}\text { Filter } \\
\text { Types }\end{array}$ & \multicolumn{5}{|c|}{ Input $\mathrm{v}_{\text {in }}$} \\
\hline $\mathrm{V}_{\text {out }}$ & $\mathrm{V}_{1}$ & $\mathrm{~V}_{2}$ & $\mathrm{~V}_{3}$ & $\mathrm{~V}_{4}$ & $\mathrm{~V}_{5}$ \\
\hline Low pass & 1 & 0 & 0 & 1 & 0 \\
\hline High pass & 0 & 1 & 0 & 1 & 0 \\
\hline Band pass & 1 & 0 & 1 & 0 & 1 \\
\hline Band reject & 1 & 1 & 0 & 1 & 0 \\
\hline All pass & 1 & 1 & 0 & 1 & 0 \\
\hline
\end{tabular}

Table 4, results the transfer function of Low-Pass (LP), HighPass (HP) Band-Pass (BP), Band-Reject (BR) and All-Pass (AP) functions. From equation (12) we can realize a different filtering function which is summarized as. 


\subsection{Universal second order Low Pass Filter}

From equation (12) transfer function of Low Pass filter is obtained by selecting input $\mathrm{V}_{1}$ and $\mathrm{V}_{4}$.

$$
\frac{V_{\text {out }}}{V_{L P}}=\frac{\frac{1}{R_{a 1}}-\frac{C_{b 1} s}{R_{b 1} C_{b 1} s+1}}{\frac{1}{R_{c}}+C_{c} s-\frac{C_{d} s}{R_{d} C_{d} s+1}}
$$

$\mathrm{N}(\mathrm{s})=\left\{\left(\mathrm{R}_{\mathrm{b} 1} \mathrm{C}_{\mathrm{b} 1} \mathrm{~s}+1\right)-\mathrm{R}_{\mathrm{a} 1} \mathrm{C}_{\mathrm{b} 1} \mathrm{~s}\right\} \times \mathrm{R}_{\mathrm{c}} \times\left(\mathrm{R}_{\mathrm{d}} \mathrm{C}_{\mathrm{d}} \mathrm{s}+1\right)$

$D(s)=\left\{\left(R_{d} C_{d} s+1\right)+R_{c} C_{c} s \times\left(R_{d} C_{d} s+1\right)-R_{c} C_{d} s\right\}$

$\times \mathrm{R}_{\mathrm{a} 1} \times\left(\mathrm{R}_{\mathrm{bl}} \mathrm{C}_{\mathrm{bl}} \mathrm{s}+1\right)$

If $\mathrm{R}_{\mathrm{bl}} \mathrm{C}_{\mathrm{bl}}=\mathrm{R}_{\mathrm{d}} \mathrm{C}_{\mathrm{d}}$, then

$\mathrm{N}(\mathrm{s})=\left(\mathrm{R}_{\mathrm{b} 1} \mathrm{R}_{\mathrm{c}} \mathrm{C}_{\mathrm{b} 1}-\mathrm{R}_{\mathrm{a} 1} \mathrm{R}_{\mathrm{c}} \mathrm{C}_{\mathrm{b} 1}\right) \mathrm{s}+\mathrm{R}_{\mathrm{c}}$

$\mathrm{D}(\mathrm{s})=\mathrm{R}_{\mathrm{a} 1} \mathrm{R}_{\mathrm{c}} \mathrm{R}_{\mathrm{d}} \mathrm{C}_{\mathrm{c}} \mathrm{C}_{\mathrm{d}} \mathrm{s}^{2}+$

$\left(\mathrm{R}_{\mathrm{a} 1} \mathrm{R}_{\mathrm{d}} \mathrm{C}_{\mathrm{d}}+\mathrm{R}_{\mathrm{a} 1} \mathrm{R}_{\mathrm{c}} \mathrm{C}_{\mathrm{c}}-\mathrm{R}_{\mathrm{a} 1} \mathrm{R}_{\mathrm{c}} \mathrm{C}_{\mathrm{d}}\right) \mathrm{s}+\mathrm{R}_{\mathrm{a} 1}$

\subsection{Universal second order High Pass Filter}

From equation (12) transfer function of High Pass filter is obtained by selecting input $\mathrm{V}_{2}$ and $\mathrm{V}_{4}$.

$$
\frac{V_{\text {out }}}{V_{H P}}=\frac{C_{a 1} s-\frac{C_{b 1} s}{R_{b 1} C_{b 1} s+1}}{\frac{1}{R_{c}}+C_{c} s-\frac{C_{d} s}{R_{d} C_{d} s+1}}
$$

$\mathrm{N}(\mathrm{s})=\left\{\left(\mathrm{C}_{\mathrm{a} 1} \mathrm{~s}\right) \times\left(\mathrm{R}_{\mathrm{b} 1} \mathrm{C}_{\mathrm{bl}} \mathrm{s}+1\right)-\mathrm{C}_{\mathrm{bl}} \mathrm{s}\right\} \times \mathrm{R}_{\mathrm{c}} \times\left(\mathrm{R}_{\mathrm{d}} \mathrm{C}_{\mathrm{d}} \mathrm{s}+1\right)$

$D(s)=\left\{\left(R_{d} C_{d} s+1\right)+R_{c} C_{c} s \times\left(R_{d} C_{d} s+1\right)-R_{c} C_{d} s\right\} \times\left(R_{b 1} C_{b 1} s+1\right)$

If $\mathrm{R}_{\mathrm{b} 1} \mathrm{C}_{\mathrm{b} 1}=\mathrm{R}_{\mathrm{d}} \mathrm{C}_{\mathrm{d}}$, then

$$
\begin{aligned}
& \mathrm{N}(\mathrm{s})=\mathrm{R}_{\mathrm{c}} \mathrm{R}_{\mathrm{b} 1} \mathrm{C}_{\mathrm{b} 1} \mathrm{C}_{\mathrm{a} 1} \mathrm{~s}^{2}+\left(\mathrm{R}_{\mathrm{c}} \mathrm{C}_{\mathrm{a} 1}-\mathrm{R}_{\mathrm{c}} \mathrm{C}_{\mathrm{b} 1}\right) \mathrm{s} \\
& \mathrm{D}(\mathrm{s})=\mathrm{R}_{\mathrm{c}} \mathrm{R}_{\mathrm{d}} \mathrm{C}_{\mathrm{c}} \mathrm{C}_{\mathrm{d}} \mathrm{s}^{2}+\left(\mathrm{R}_{\mathrm{d}} \mathrm{C}_{\mathrm{d}}+\mathrm{R}_{\mathrm{c}} \mathrm{C}_{\mathrm{c}}-\mathrm{R}_{\mathrm{c}} \mathrm{C}_{\mathrm{d}}\right) \mathrm{s}+1
\end{aligned}
$$

\subsection{Universal second order Band Pass Filter}

From equation (12) transfer function of Band Pass filter is obtained by selecting input $\mathrm{V}_{1}, \mathrm{~V}_{3}$ and $\mathrm{V}_{5}$.

$$
\frac{V_{\text {out }}}{V_{B P}}=\frac{\frac{1}{R_{a 1}}+\frac{C_{a 2} s}{R_{a 2} C_{a 2} s+1}-\frac{1}{R_{b 2}}}{\frac{1}{R_{c}}+C_{c} s-\frac{C_{d} s}{R_{d} C_{d} s+1}}
$$

$\mathrm{N}(\mathrm{s})=\left\{\mathrm{R}_{\mathrm{b} 2} \times\left(\mathrm{R}_{\mathrm{a} 2} \mathrm{C}_{\mathrm{a} 2} \mathrm{~s}+1\right)+\mathrm{R}_{\mathrm{a} 1} \mathrm{R}_{\mathrm{b} 2} \mathrm{C}_{\mathrm{a} 2} \mathrm{~s}-\mathrm{R}_{\mathrm{a} 1} \mathrm{R}_{\mathrm{a} 2} \mathrm{C}_{\mathrm{a} 2} \mathrm{~s}-\mathrm{R}_{\mathrm{a} 1}\right\}$

$\times \operatorname{Rc} \times\left(\mathrm{R}_{\mathrm{d}} \mathrm{C}_{\mathrm{d}} \mathrm{s}+1\right)$

$D(s)=\left\{\left(R_{d} C_{d} s+1\right)+R_{c} C_{c} s \times\left(R_{d} C_{d} s+1\right)-R_{c} C_{d} s\right\}$

$\times \mathrm{R}_{\mathrm{a} 1} \mathrm{R}_{\mathrm{b} 2} \times\left(\mathrm{R}_{\mathrm{a} 2} \mathrm{C}_{\mathrm{a} 2} \mathrm{~s}+1\right)$

If $\mathrm{R}_{\mathrm{a} 2} \mathrm{C}_{\mathrm{a} 2}=\mathrm{R}_{\mathrm{d}} \mathrm{C}_{\mathrm{d}}$, then

$\mathrm{N}(\mathrm{s})=\left(\mathrm{R}_{\mathrm{c}} \mathrm{R}_{\mathrm{b} 2} \mathrm{R}_{\mathrm{a} 2} \mathrm{C}_{\mathrm{a} 2}+\mathrm{R}_{\mathrm{c}} \mathrm{R}_{\mathrm{a} 1} \mathrm{R}_{\mathrm{b} 2} \mathrm{C}_{\mathrm{a} 2}-\mathrm{R}_{\mathrm{c}} \mathrm{R}_{\mathrm{a} 1} \mathrm{R}_{\mathrm{a} 2} \mathrm{C}_{\mathrm{a} 2}\right) \mathrm{s}$

$+\left(\mathrm{R}_{\mathrm{c}} \mathrm{R}_{\mathrm{b} 2}-\mathrm{R}_{\mathrm{c}} \mathrm{R}_{\mathrm{a} 1}\right)$

$\mathrm{D}(\mathrm{s})=\mathrm{R}_{\mathrm{a} 1} \mathrm{R}_{\mathrm{b} 2} \mathrm{R}_{\mathrm{c}} \mathrm{R}_{\mathrm{d}} \mathrm{C}_{\mathrm{c}} \mathrm{C}_{\mathrm{d}} \mathrm{s}^{2}+$

$\left(\mathrm{R}_{\mathrm{a} 1} \mathrm{R}_{\mathrm{b} 2} \mathrm{R}_{\mathrm{d}} \mathrm{C}_{\mathrm{d}}+\mathrm{R}_{\mathrm{a} 1} \mathrm{R}_{\mathrm{b} 2} \mathrm{R}_{\mathrm{c}} \mathrm{C}_{\mathrm{c}}-\mathrm{R}_{\mathrm{a} 1} \mathrm{R}_{\mathrm{b} 2} \mathrm{R}_{\mathrm{c}} \mathrm{C}_{\mathrm{d}}\right) \mathrm{s}+\mathrm{R}_{\mathrm{a} 1} \mathrm{R}_{\mathrm{b} 2}$
2.4 Universal second order Band Reject Filter

From equation (12) transfer function of Band Reject filter is obtained by selecting input $V_{1}, V_{2}$ and $V_{4}$.

$$
\frac{V_{\text {out }}}{V_{\text {BRF }}}=\frac{\frac{1}{R_{a 1}}+C_{a 1} s-\frac{C_{b 1} s}{R_{b 1} C_{b 1} s+1}}{\frac{1}{R_{c}}+C_{c} s-\frac{C_{d} s}{R_{d} C_{d} s+1}}
$$

$\mathrm{N}(\mathrm{s})=\left\{\left(\mathrm{R}_{\mathrm{bl}} \mathrm{C}_{\mathrm{bl}} \mathrm{s}+1\right)+\mathrm{R}_{\mathrm{a} 1} \mathrm{C}_{\mathrm{a} 1} \mathrm{~s} \times\left(\mathrm{R}_{\mathrm{b} 1} \mathrm{C}_{\mathrm{a} 1} \mathrm{~s}+1\right)-\mathrm{R}_{\mathrm{a} 1} \mathrm{C}_{\mathrm{bl}} \mathrm{s}\right\}$

$\times \mathrm{R}_{\mathrm{c}} \times\left(\mathrm{R}_{\mathrm{d}} \mathrm{C}_{\mathrm{d}} \mathrm{s}+1\right)$

$D(s)=\left\{\left(R_{d} C_{d} s+1\right)+R_{c} C_{c} s \times\left(R_{d} C_{d} s+1\right)-R_{c} C_{d} s\right\}$

$\times \mathrm{R}_{\mathrm{a} 1} \times\left(\mathrm{R}_{\mathrm{bl}} \mathrm{C}_{\mathrm{b} 1} \mathrm{~s}+1\right)$

If $\mathrm{R}_{\mathrm{bl}} \mathrm{C}_{\mathrm{bl}}=\mathrm{R}_{\mathrm{d}} \mathrm{C}_{\mathrm{d}}$, then

$\mathrm{N}(\mathrm{s})=\mathrm{R}_{\mathrm{a} 1} \mathrm{R}_{\mathrm{c}} \mathrm{R}_{\mathrm{bl}} \mathrm{C}_{\mathrm{bl}} \mathrm{s}^{2}+\left(\mathrm{R}_{\mathrm{c}} \mathrm{R}_{\mathrm{bl}} \mathrm{C}_{\mathrm{b} 1}+\mathrm{R}_{\mathrm{c}} \mathrm{R}_{\mathrm{a} 1} \mathrm{C}_{\mathrm{a} 1}-\mathrm{R}_{\mathrm{c}} \mathrm{R}_{\mathrm{a} 1} \mathrm{C}_{\mathrm{b} 1}\right) \mathrm{s}$

$+\mathrm{R}_{\mathrm{c}}$

$\mathrm{D}(\mathrm{s})=\mathrm{R}_{\mathrm{a} 1} \mathrm{R}_{\mathrm{c}} \mathrm{R}_{\mathrm{d}} \mathrm{C}_{\mathrm{c}} \mathrm{C}_{\mathrm{d}} \mathrm{s}^{2}+\left(\mathrm{R}_{\mathrm{al}} \mathrm{R}_{\mathrm{d}} \mathrm{C}_{\mathrm{d}}+\mathrm{R}_{\mathrm{a} 1} \mathrm{R}_{\mathrm{c}} \mathrm{C}_{\mathrm{c}}-\mathrm{R}_{\mathrm{al}} \mathrm{R}_{\mathrm{c}} \mathrm{C}_{\mathrm{d}}\right) \mathrm{s}$

$+\mathrm{R}_{\mathrm{a} 1}$

2.5 Universal second order All Pass Filter

From equation (12) transfer function of All Pass filter is obtained by selecting input $V_{1}, V_{2}$ and $V_{4}$.

$$
\frac{V_{\text {out }}}{V_{A P}}=\frac{\frac{1}{R_{a 1}}+C_{a 1} s-\frac{C_{b 1} s}{R_{b 1} C_{b 1} s+1}}{\frac{1}{R_{c}}+C_{c} s-\frac{C_{d} s}{R_{d} C_{d} s+1}}
$$

$\mathrm{N}(\mathrm{s})=\left\{\left(\mathrm{R}_{\mathrm{bl}} \mathrm{C}_{\mathrm{b} 1} \mathrm{~s}+1\right)+\mathrm{R}_{\mathrm{a} 1} \mathrm{C}_{\mathrm{a} 1} \mathrm{~s} \times\left(\mathrm{R}_{\mathrm{b} 1} \mathrm{C}_{\mathrm{a} 1} \mathrm{~s}+1\right)-\mathrm{R}_{\mathrm{a} 1} \mathrm{C}_{\mathrm{b} 1} \mathrm{~s}\right\}$ $\times \mathrm{R}_{\mathrm{c}} \times\left(\mathrm{R}_{\mathrm{d}} \mathrm{C}_{\mathrm{d}} \mathrm{s}+1\right)$

$D(s)=\left\{\left(R_{d} C_{d} s+1\right)+R_{c} C_{c} s \times\left(R_{d} C_{d} s+1\right)-R_{c} C_{d} s\right\}$

$\times \mathrm{R}_{\mathrm{a} 1} \times\left(\mathrm{R}_{\mathrm{bl}} \mathrm{C}_{\mathrm{bl}} \mathrm{s}+1\right)$

If $\mathrm{R}_{\mathrm{bl}} \mathrm{C}_{\mathrm{bl}}=\mathrm{R}_{\mathrm{d}} \mathrm{C}_{\mathrm{d}}$, then

$\mathrm{N}(\mathrm{s})=\mathrm{R}_{\mathrm{a} 1} \mathrm{R}_{\mathrm{c}} \mathrm{R}_{\mathrm{bl}} \mathrm{C}_{\mathrm{bl}} \mathrm{s}^{2}+$

$\left(\mathrm{R}_{\mathrm{c}} \mathrm{R}_{\mathrm{b} 1} \mathrm{C}_{\mathrm{b} 1}+\mathrm{R}_{\mathrm{c}} \mathrm{R}_{\mathrm{a} 1} \mathrm{C}_{\mathrm{a} 1}-\mathrm{R}_{\mathrm{c}} \mathrm{R}_{\mathrm{a} 1} \mathrm{C}_{\mathrm{b} 1}\right) \mathrm{s}+\mathrm{R}_{\mathrm{c}}$

$\mathrm{D}(\mathrm{s})=\mathrm{R}_{\mathrm{a} 1} \mathrm{R}_{\mathrm{c}} \mathrm{R}_{\mathrm{d}} \mathrm{C}_{\mathrm{c}} \mathrm{C}_{\mathrm{d}} \mathrm{s}^{2}+$

$\left(\mathrm{R}_{\mathrm{a} 1} \mathrm{R}_{\mathrm{d}} \mathrm{C}_{\mathrm{d}}+\mathrm{R}_{\mathrm{a} 1} \mathrm{R}_{\mathrm{c}} \mathrm{C}_{\mathrm{c}}-\mathrm{R}_{\mathrm{a} 1} \mathrm{R}_{\mathrm{c}} \mathrm{C}_{\mathrm{d}}\right) \mathrm{s}+\mathrm{R}_{\mathrm{a} 1}$

All filter realization must have some condition. The condition for realization of low pass, high pass, band pass, band reject and all pass filters are summarised in Table 5 .

The natural frequency and quality factor of the designed circuit for low pass, high pass, band pass, band reject and all pass filter can be obtained as

$$
\begin{gathered}
\omega_{0}=\frac{1}{\sqrt{\mathrm{R}_{\mathrm{c}} \mathrm{R}_{\mathrm{d}} \mathrm{C}_{\mathrm{c}} \mathrm{C}_{\mathrm{d}}}} \\
\mathrm{Q}_{\mathrm{o}}=\frac{\sqrt{\mathrm{R}_{\mathrm{c}} \mathrm{R}_{\mathrm{d}} \mathrm{C}_{\mathrm{c}} \mathrm{C}_{\mathrm{d}}}}{\left(\mathrm{R}_{\mathrm{d}} \mathrm{C}_{\mathrm{d}}+\mathrm{R}_{\mathrm{c}} \mathrm{C}_{\mathrm{c}}+\mathrm{R}_{\mathrm{c}} \mathrm{C}_{\mathrm{d}}\right)}
\end{gathered}
$$

and the sensitivity of $\omega_{0}$ and $Q_{0}$ with respect to passive elements may be expressed as 


$$
\begin{gathered}
\mathrm{S}_{\mathrm{R}_{\mathrm{c}}}^{\omega_{\mathrm{O}}}=\mathrm{S}_{\mathrm{R}_{\mathrm{d}}}^{\omega_{\mathrm{O}}}=\mathrm{S}_{\mathrm{Cc}}^{\omega_{\mathrm{O}}}=\mathrm{S}_{\mathrm{C}_{\mathrm{d}}}^{\omega_{\mathrm{o}}}=-\frac{1}{2} \text { and } \\
\mathrm{S}_{\mathrm{R}_{\mathrm{c}}}^{\mathrm{Q}}=\mathrm{S}_{\mathrm{R}_{\mathrm{d}}}^{\mathrm{Q}}=\mathrm{S}_{\mathrm{Cc}}^{\mathrm{Q}}=\mathrm{S}_{\mathrm{C}_{\mathrm{d}}}^{\mathrm{Q}}=-\frac{1}{2}
\end{gathered}
$$

It proclaims that the designed circuit gives low sensitivity.

\section{TABLE 5: CONDITION OF REALIZATION OF EACH FILTER.}

\begin{tabular}{|l|l|}
\hline $\begin{array}{l}\text { Filter } \\
\text { response }\end{array}$ & \multicolumn{1}{c|}{ Condition } \\
\hline Low Pass & $\mathrm{R}_{\mathrm{b} 1} \mathrm{C}_{\mathrm{b} 1}=\mathrm{R}_{\mathrm{d}} \mathrm{C}_{\mathrm{d}}, \mathrm{R}_{\mathrm{b} 1} \mathrm{C}_{\mathrm{b} 1} \mathrm{R}_{\mathrm{c}}=\mathrm{R}_{\mathrm{a} 1} \mathrm{C}_{\mathrm{b} 1} \mathrm{R}_{\mathrm{c}}$ \\
\hline High Pass & $\mathrm{R}_{\mathrm{b} 1} \mathrm{C}_{\mathrm{b} 1}=\mathrm{R}_{\mathrm{d}} \mathrm{C}_{\mathrm{d},}, \mathrm{R}_{\mathrm{c}} \mathrm{Ca} 1=\mathrm{R}_{\mathrm{c}} \mathrm{C}_{\mathrm{b} 1}$ \\
\hline Band Pass & $\mathrm{R}_{\mathrm{a} 2} \mathrm{C}_{\mathrm{a} 2}=\mathrm{R}_{\mathrm{d}} \mathrm{C}_{\mathrm{d},}, \mathrm{R}_{\mathrm{c}} \mathrm{R}_{\mathrm{b} 2}=\mathrm{R}_{\mathrm{c}} \mathrm{R}_{\mathrm{a} 1}$ \\
\hline Band Reject & $\begin{array}{l}\mathrm{R}_{\mathrm{b} 1} \mathrm{C}_{\mathrm{b} 1}=\mathrm{R}_{\mathrm{d}} \mathrm{C}_{\mathrm{d}}, \mathrm{R}_{\mathrm{c}} \\
\mathrm{R}_{\mathrm{b} 1} \mathrm{C}_{\mathrm{b} 1}+\mathrm{R}_{\mathrm{c}} \mathrm{R}_{\mathrm{a} 1} \mathrm{C}_{\mathrm{a} 1}=\mathrm{R}_{\mathrm{c}} \mathrm{R}_{\mathrm{a} 1} \mathrm{C}_{\mathrm{b} 1}\end{array}$ \\
\hline All pass & $\begin{array}{l}\mathrm{R}_{\mathrm{b} 1} \mathrm{C}_{\mathrm{b} 1}=\mathrm{R}_{\mathrm{d}} \mathrm{C}_{\mathrm{d}}, \\
\mathrm{R}_{\mathrm{a} 1} \mathrm{R}_{\mathrm{c}} \mathrm{C}_{\mathrm{a} 1} \mathrm{R}_{\mathrm{b} 1}=\mathrm{R}_{\mathrm{a} 1} \mathrm{R}_{\mathrm{c}} \mathrm{C}_{\mathrm{c}} \mathrm{R}_{\mathrm{d}} \mathrm{C}_{\mathrm{d}}, \\
-\left(\mathrm{R}_{\mathrm{c}} \mathrm{R}_{\mathrm{d}} \mathrm{C}_{\mathrm{d}}+\mathrm{R}_{\mathrm{c}} \mathrm{R}_{\mathrm{a} 1} \mathrm{C}_{\mathrm{a} 1}-\mathrm{R}_{\mathrm{c}} \mathrm{R}_{\mathrm{a} 1} \mathrm{C}_{\mathrm{b} 1}\right) \\
=\left(\mathrm{R}_{\mathrm{a} 1} \mathrm{R}_{\mathrm{d}} \mathrm{C}_{\mathrm{d}}+\mathrm{R}_{\mathrm{a} 1} \mathrm{R}_{\mathrm{c}} \mathrm{C}_{\mathrm{c}}-\mathrm{R}_{\mathrm{a} 1} \mathrm{R}_{\mathrm{c}} \mathrm{C}_{\mathrm{d}}\right), \mathrm{R}_{\mathrm{c}}=\mathrm{R}_{\mathrm{a} 1}\end{array}$ \\
\hline
\end{tabular}

\section{NON-IDEALITY ANALYSIS Of OTRA}

Normally the trans-resistance gain is assumed to infinity for filter designing. However, practically trans-resistance gain $\left(R_{m}\right)$ is a frequency dependent finite value. Considering a single pole model for the trans-resistance gain can be approximately given in terms of high frequencies as

$$
\mathrm{R}_{\mathrm{m}} \approx \frac{1}{\mathrm{C}_{\mathrm{p}} \mathrm{s}}
$$

where,

$$
\mathrm{Cp}=\frac{1}{\omega_{\mathrm{O}} \mathrm{R}_{\mathrm{O}}}
$$

where, $\omega_{0}$ is the pole frequency and $R_{0}$ is dc transresistance gain.

Taking non-ideality effect of OTRA the transfer function in equation (2) modifies to

$$
\frac{\mathrm{N}(\mathrm{s})}{\mathrm{D}(\mathrm{s})}=\frac{\mathrm{Y}_{1} \mathrm{~V}_{1}+\mathrm{Y}_{2} \mathrm{~V}_{2}+\mathrm{Y}_{3} \mathrm{~V}_{3}-\mathrm{Y}_{4} \mathrm{~V}_{4}-\mathrm{Y}_{5} \mathrm{~V} 5}{\mathrm{Y}_{6}+\mathrm{Y}_{7}-\mathrm{Y}_{8}+\mathrm{sC}_{\mathrm{p}}}
$$

If the denominator of equation(2) modifies as equation(33) or in other words admittances $\mathrm{Y}_{6} \mathrm{Y}_{7}$ and $\mathrm{Y}_{8}$ contain a parallel capacitor, this result a complete self compensation [10]. In our designed circuits, $\mathrm{Y}_{7}$ contains a parallel capacitor branch , hence the designed filters taking the magnitude of $\mathrm{Cp}$ into consideration. In this way, the effect of $\mathrm{Cp}$ can be absorbed in capacitance $\mathrm{Y}_{7}$ without using additional elements and achieving complete self compensation [10].

\section{Simulation RESUlT}

The designed $0.5 \mu \mathrm{m}$ Complementary metal oxide semiconductor technology based internal circuit of operational trans-resistance amplifier as shown in Figure 1[5], with dc power supply voltages $V_{\mathrm{DD}}=-V_{\mathrm{SS}}=1.5 \mathrm{volt}$ and bias voltage $V_{\mathrm{B}}=-0.5$ volt. The simulations are performed using ORCAD 10.5 circuit simulator based on $0.5 \mu \mathrm{m}$ Complementary metal oxide semiconductor technology. The filter is designed for a natural frequency ( $3 \mathrm{~dB}$ frequency) of $\mathrm{f}_{0}=100 \mathrm{kHz}$. Comparative simulated and theoretical result of the Butterworth, Chebyshev and Bessel filters, followed by five sections describing the most common filter response: low pass, high pass, band pass, band reject and all pass filters are shown in Figure 5. The Butterworth gives flat frequency response in the pass band and its stop band attenuates with $-40 \mathrm{~dB} /$ decade as given in Figure 5. The Chebyshev gives a sharper roll off with comparison to Butterworth and Bessel filters, but allowing distortion in form of ripple in the frequency response shown in Figure 5. As the roll-off more sharper, the ripple become increases, so trade-off between these two parameters observed in the Chebyshev response. The Bessel filters gives constant group delay for wide frequency range because it shows a linear phase response over a wide frequency range with comparison to Butterworth and Chebyshev filters. Figure 6 shown the comparison result of phase response of Butterworth, Chebyshev and Bessel all pass filter.

To verify the output quality, total harmonic distortion is obtained for low pass Butterworth, Chebyshev and Bessel filters as shown in Figure 7. It is found that the output distortion is very small and it is $5 \%$ up to 4 volt[1]. It proclaim that the output of filters are very good quality and dynamic range is high. The simulated results verify with the theoretical results, shown in Figure 5. A comparative study for the second order universal filter is shown in Table 6 .

TABLE 6. COMPARISION OF AVAILABLE SECOND ORDER UNIVERSAL FILTERS

\begin{tabular}{|c|c|c|c|c|c|c|}
\hline $\begin{array}{c}\text { Reference } \\
\text { Number }\end{array}$ & Active element & $\begin{array}{c}\text { Required } \\
\text { active } \\
\text { elements }\end{array}$ & $\begin{array}{c}\text { Required } \\
\text { capacitors }\end{array}$ & $\begin{array}{c}\text { Required } \\
\text { resistors }\end{array}$ & $\begin{array}{c}\text { Requirement of } \\
\text { Inbuilt tunability }\end{array}$ & $\begin{array}{c}\text { Requirement of } \\
\text { change the } \\
\text { hardware to } \\
\text { change filter } \\
\text { types }\end{array}$ \\
\hline 17 & DVCCTA & 1 & 2 & 1 & Yes & Yes \\
\hline 18 & CCCII & 4 & 2 & No & Yes & No \\
\hline 19 & CDTA & 2 & 2 & 2 & Yes & Yes \\
\hline 20 & CCII & 3 & 2 & 2 & No & Yes \\
\hline Proposed & OTRA & 1 & 4 & 4 & No & Yes \\
\hline
\end{tabular}



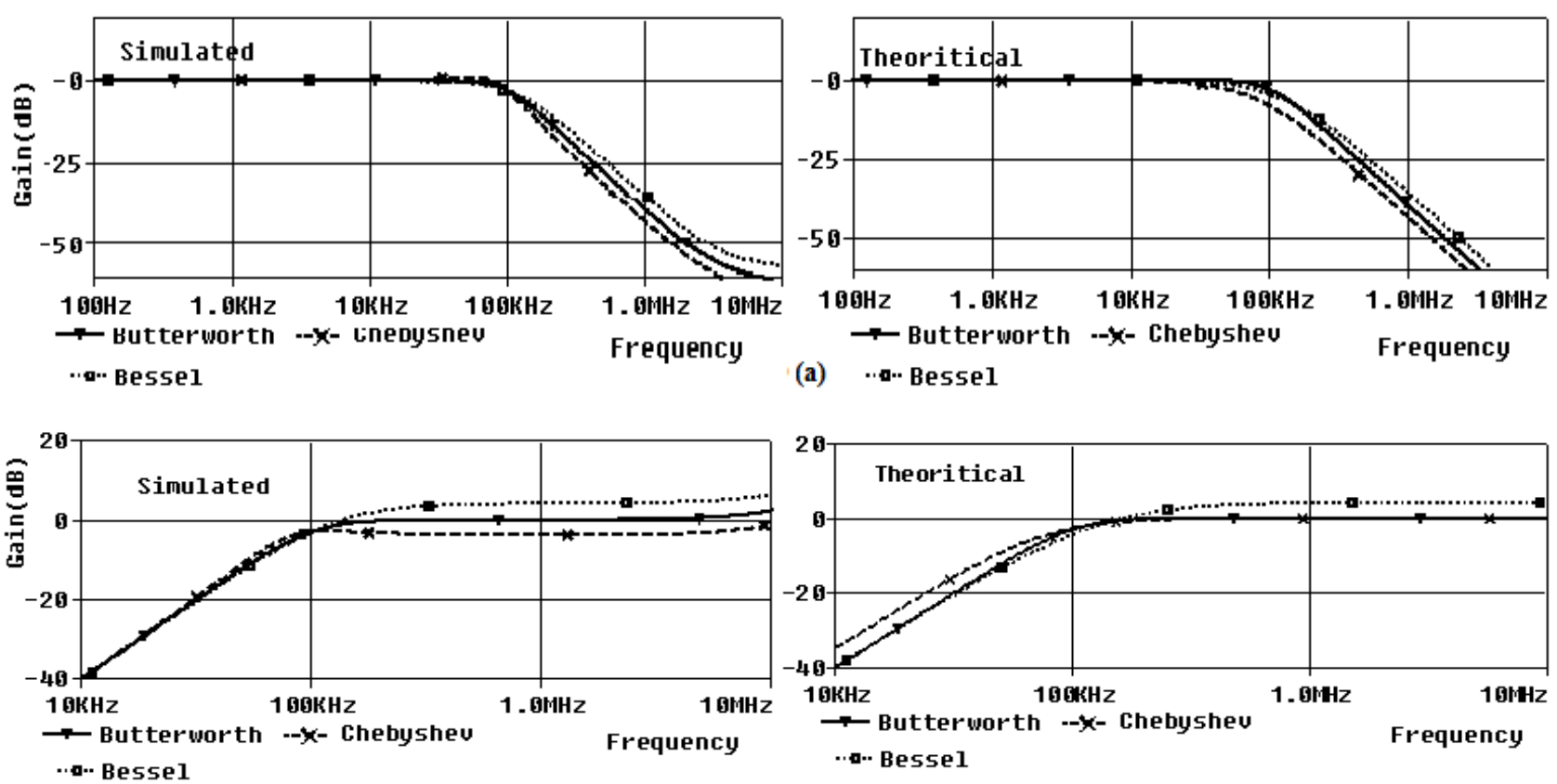

(b)
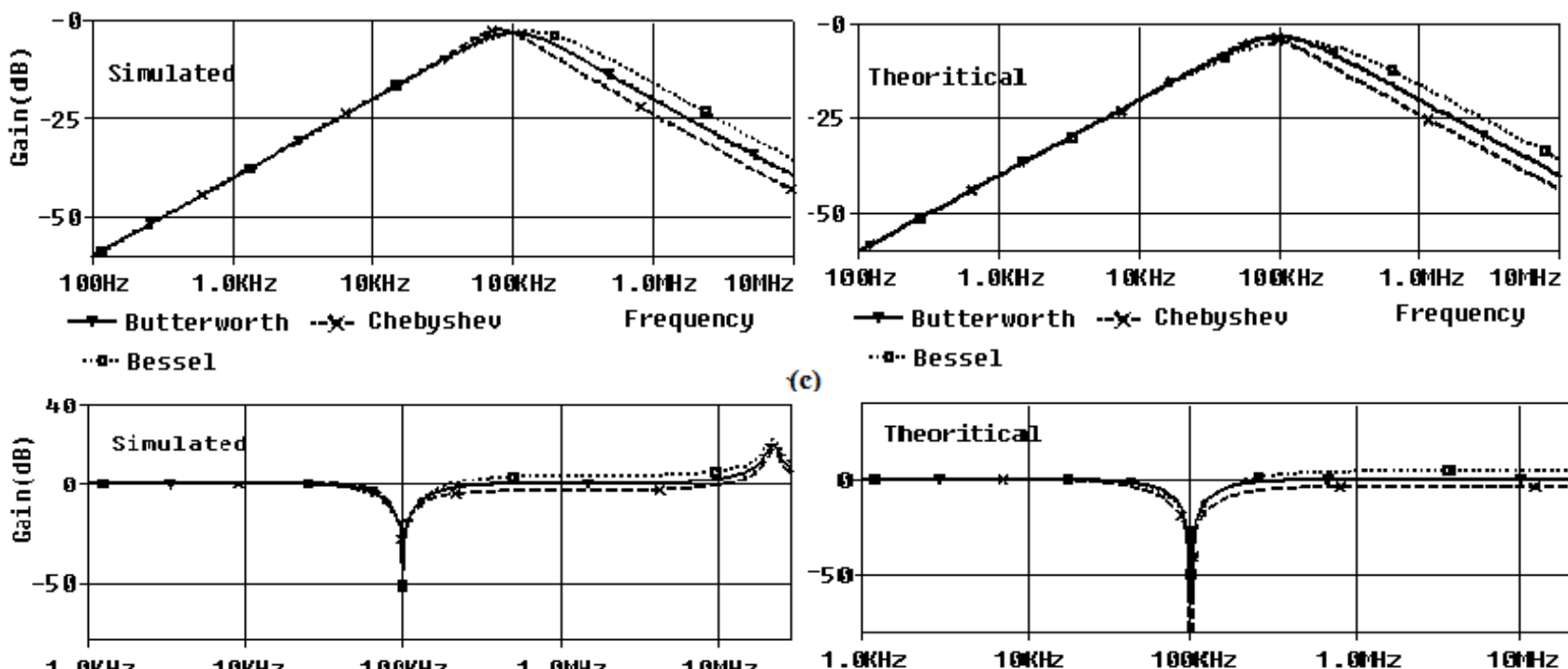

(c)
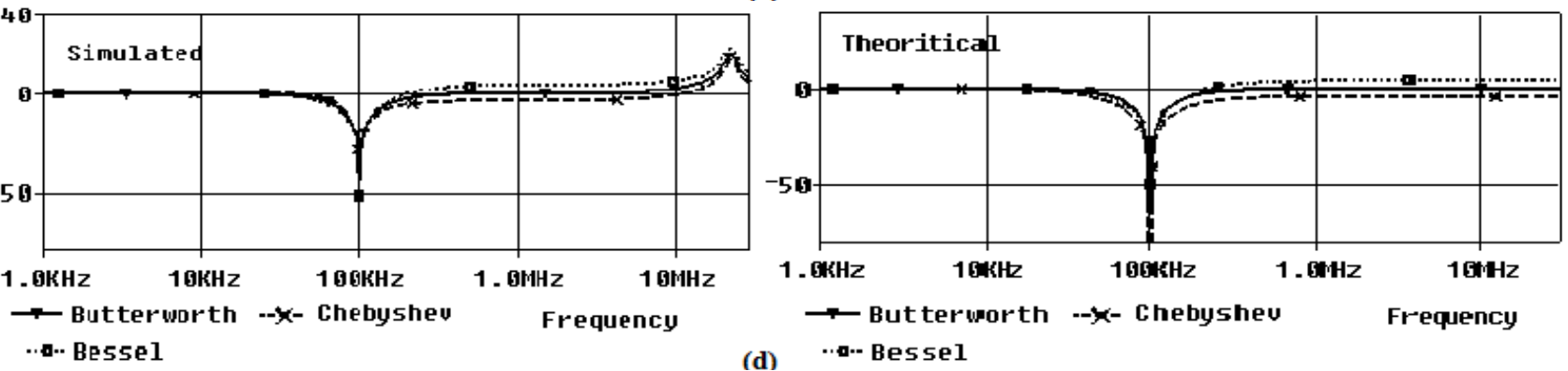

(d)

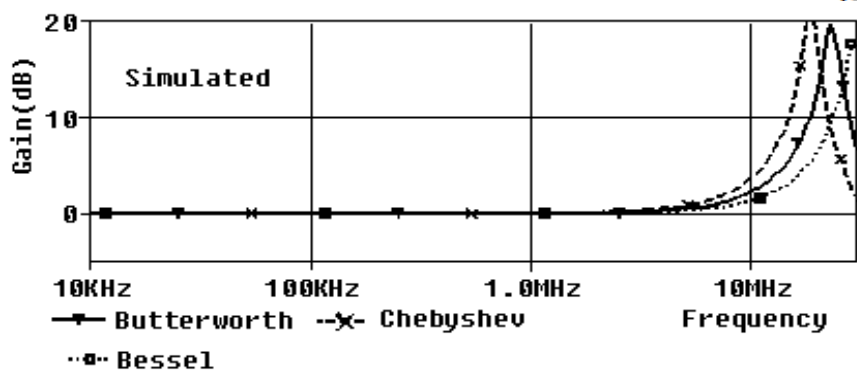

$\rightarrow$ But terworth $\rightarrow$ - chebyshev Fessel
..- Besuency

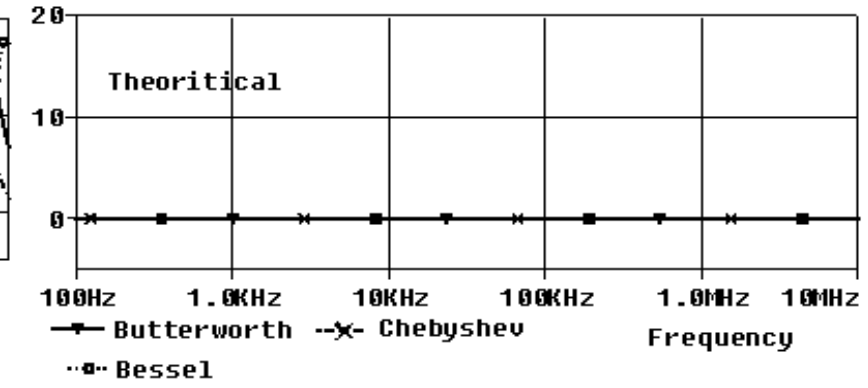

(e)

Fig.5. Comprative simulated and theoritical result of universal second order Butterworth, Chebyshev and Bessel Filters .(a) low pass (b) high pass (c) band pass (d) band Reject (e) all pass. 


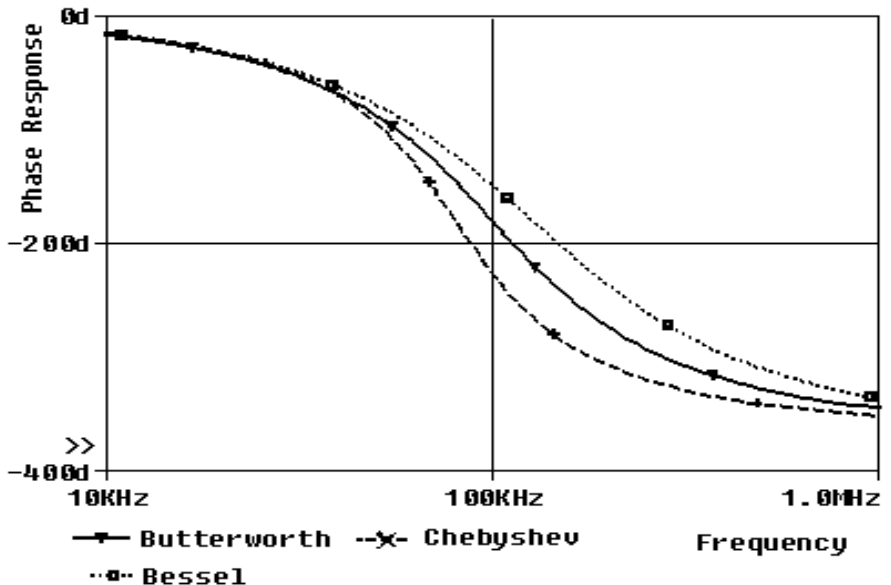

Fig.6. Comparison result of phase response of Butterworth, Chebyshev and Bessel all pass filter.

\section{CONCLUSION}

In this paper, a New $0.5 \mu \mathrm{m}$ CMOS-Based OTRA Based voltage mode second-order universal Butterworth, Chebyshev and Bessel filters Structure is presented, the proposed filter structure uses a single OTRA as an active component, It makes this designed work economical one. Also it have not required in built tunability of filter parameters. The proposed circuit offers low sensitivity. The theoretical result is quite agreed with simulated result.

\section{REFERENCES}

[1]. E. S. Erdogan and O. Cicekoglu, "New Current Mode Specia Function Continuous- Time Active Filters Employing Only OTAs and OPAMPs", International Journal of Electronics, Vol. 91, pp. 345-359, 2004.

[2]. R. Šotner and J. Slezák, "Current-Controlled Current Mode Universal Biquad Employing Multi-Output Transconductors", Radioengineering, vol. 18, no. 3, pp. 285-294, 2009.

[3]. C. Toumazou, and F.J. Ledgey, "Universal Active Filter Using Current Conveyors", Electronics Letters, Vol. 22, pp. 662-664. 1986.

[4]. A. U. Keskin and E. Hancioglu, "Current mode multifunction filter using two Current Differencing Buffer Amplifiers," International Journal of Electronics and Communications, vol. 59, no. 8, pp. 495-498, 2005

[5]. K. N. Salama and A. M. Soliman, "CMOS OTRA for analog signa processing applications", Microelectronics Journal, Vol. 3 pp. 236 $245,1999$.

[6]. H. Mostafa "A modified CMOS realization of the operational transresistance amplifier (OTRA)," Frequenz, vol. 60, no. 2-4, pp. 70-76, 2006.

[7]. J. J. Chen and C. Chen, "Operational transresistance amplifier using CMOS technology", Electronics Letters, Vol. 8, pp. 2087 2088, 1992

[8]. J. J. Chen, H.W. Tsao and W. Chui, "Parasitic-capacitanceinsensitive current-mode filters using operational transresistance

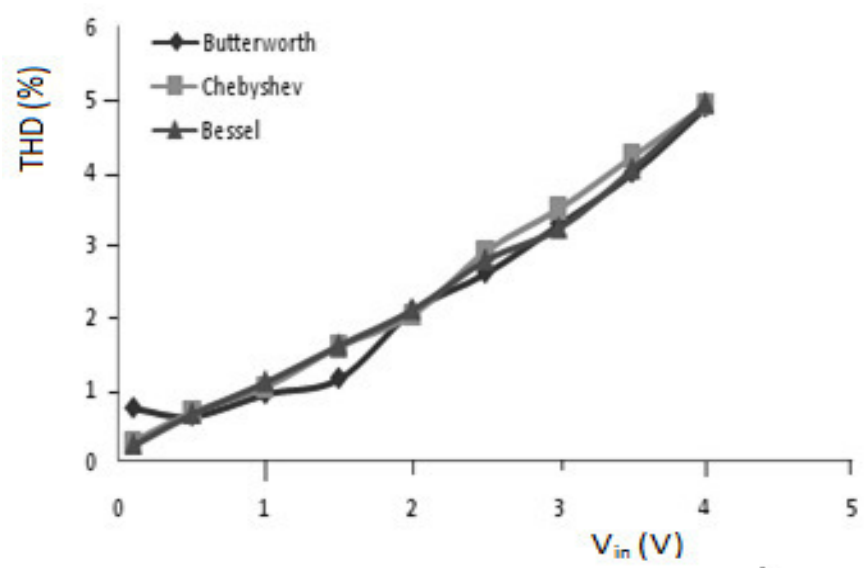

Fig.7. THD Variation with respect to input voltage.

amplifier", IEE Proceedings Part G: Circuits, Devices and Systems, Vol. 142, pp. 186-192, 1995.

[9]. K. N. Salama, and A.M. Soliman, "Universal filters using the OTRAs", AEU-International Journal of Electronics and Communications, Vol. 53, pp. 49-52, 1999.

[10]. U. Cam, "A novel single-resistance-controlled snusoidal oscillator employing single operational transresistancenamplifier", Analog Integrated Circuits and Signal Processing, Vol. 32, pp. 184-186, 2002.

[11]. U. Cam and O. Cicekoglu, "Novel transimpedance type first-order all-pass filter using single OTRA", AEU International Journal of Electronics \& Communications, Vol. 58, pp. 296-298, 2004

[12]. R. Senani, "KHN-equivalent bi-quad using current conveyors", Electronics Letters, Vol. 31, pp. 625-628, 1995.

[13]. S. Shah, "Design of KHN bi-quad using operational transconductance amplifier", The $200245^{\text {th }}$ Midwest Symposium on Circuit and Systems, Vol. 1, pp. 48-51, 2002.

[14]. A.M. Soliman, "Voltage mode and current mode Tow Thomas biquadratic filters using ICCII", International Journal of Circuit Theory and Applications, Vol. 35, pp. 462-466, 2007.

[15]. P.E. Fleischer and J. Tow, "Design formulas for bi-quad filters using three operational amplifiers", Proceedings of the IEEE, Proceeding Letters, Vol. 61, pp. 662-663, 1973.

[16]. S. Kilinc and U. Cam, "Realization of $\mathrm{n}^{\text {th }}$ Order Voltage Transfer Function using a Single Operational Transresistance Amplifier", ETRI Journal, vol. 27, no. 5, pp. 647-650. 2005.

[17]. N. Pandey and S. K. Paul "VM and CM Universal Filters Based on Single DVCCTA", Hindawi Publishing Corporation Active and Passive Electronic Components, Vol. 2011.

[18]. C. Wang, H. Liu and Yan Zhao, "A New Current-Mode CurrentControlled Universal Filter Based on CCCII $( \pm)$ ", Circuits Syst Signal Process, pp.673-682, 2008.

[19]. N. A. Shah E. M. Quadri E. Syed and Z. Iqbal, "CDTA based universal transadmittance filter", Analog Integr Circ Sig Process , pp. $65-69.2007$

[20]. J. W. Horng, "High Order Current-Mode and Trans-impedance Mode Universal Filters with Multiple Inputs and Two Outputs Using MOCCIIs," Radioengineering, vol. 8, no. 4, pp. 537-543, 2009 